\title{
Hyperonic nuclear matter in Brueckner theory
}

\author{
H.-J. Schulze, M. Baldo, and U. Lombardo \\ Sezione INFN, Università di Catania, Corso Italia 57, I-95129 Catania, Italy \\ J. Cugnon and A. Lejeune \\ Institut de Physique B5, Université de Liège, B-4000 Liège 1, Belgium
}

(Received 3 July 1997)

\begin{abstract}
We determine in an extended Brueckner-Hartree-Fock formalism self-consistent single-particle potentials of nucleons, lambda, and sigma hyperons for a system consisting of symmetric nuclear matter and lambda hyperons of uniform densities $\rho_{N}$ and $\rho_{\Lambda}$, respectively. The binding energy per baryon of this system is discussed and its maximum strangeness content preserving binding is evaluated. The results are used to introduce a hyperonic symmetry energy term in a generalized mass formula for multistrange hypernuclei. [S0556-2813(98)01101-7]
\end{abstract}

PACS number(s): 21.65.+f, 21.80.+a, 24.10.Cn

\section{INTRODUCTION}

There is an increasing interest in exploring nuclear systems with strangeness, especially multistrange nuclear systems, i.e., those containing several strange particles. Besides simple hypernuclei, which have been studied for a long time already [1], a few doubly strange hypernuclei have been identified [2]. Recently, the production of hyperfragments in relativistic heavy-ion collisions [3] has received more attention. Furthermore, the core of neutron stars, which is the object of theoretical investigations [4], may contain a high fraction of hyperons. The strangeness content of nuclear systems is regarded more and more as another degree of freedom, in similarity to the $N / Z$ ratio.

Theoretical investigations of these systems have a limited reliability, mainly due to the fragmentary knowledge of the hyperon-nucleon and especially hyperon-hyperon interactions. The Nijmegen [5] and Jülich [6] groups have exploited the scarce existing measurements to build detailed mesonexchange potentials. Although both potentials describe the hyperon-nucleon scattering data reasonably well, they give different predictions for many-body systems, partly because they assume different exchanges, but mainly because the existing data do not constrain the potentials sufficiently. The forthcoming experiments at BNL and KEK [7] will, for the first time in about 30 years, yield fresh experimental information that will hopefully improve the reliability of available hyperon-nucleon and hyperon-hyperon potentials.

All the above-mentioned applications involve the effects of the hyperon-nucleon (and hyperon-hyperon) interaction in a more or less dense baryonic medium. In this article we present the state of the art of hypernuclear matter calculations in an extended Brueckner-Hartree-Fock (BHF) formalism with the Paris nucleon-nucleon [8] and the Nijmegen soft-core hyperon-nucleon [5] potentials. This work is an extension of a preliminary study [9] that was restricted to a fixed nucleon density $\rho_{N}=\rho_{0}=0.17 \mathrm{fm}^{-3}$. We consider now varying lambda as well as nucleon densities. We calculate the binding energy and the nucleon, lambda, and sigma mean fields for such a system. We also determine the hyperonic symmetry energy in a generalized mass formula [10] for finite systems composed of nucleons and lambda hyperons.

Of course, multistrange systems, especially those that can be created in heavy-ion collisions, may contain other hyperons. However, for not too large hyperonic densities, it is expected that $\Xi$ particles decay quickly through the $N$ $+\Xi \rightarrow \Lambda+\Lambda$ process. Similarly, in the same conditions, the strong interaction process $\Sigma+N \rightarrow \Lambda+N$ is always possible, and sigma hyperons rapidly disappear from the medium. Therefore, hypermatter should appear as a mixture of nucleons and lambda particles for a relatively long time, determined basically by the characteristic weak decay time of lambda particles inside the medium. The latter is so long that the system can be considered as equilibrated with respect to the strong interactions. For large hyperonic densities, the strong decays mentioned above may be hindered by the Pauli blocking inside the Fermi sea of $\Lambda$ particles $[10,11]$. In that case, the addition of $\Sigma$ and $\Xi$ Fermi seas would be mandatory.

\section{FORMALISM}

We consider in this article the properties of an infinite system composed of nucleons (protons and neutrons of same proportion) and lambda hyperons with moderate strangeness fraction $\rho_{\Lambda} / \rho_{N} \lesssim 0.3$. This allows us to disregard $\Sigma$ and $\Xi$ components as mentioned above. However, we do consider $\Sigma$ hyperons in the intermediate states, as it is well known that the $\Lambda N$ and $\Sigma N$ channels are strongly coupled. A related reason for the restriction to a small hyperon fraction is the fact that quantitative properties (potentials) for the hyperonhyperon interactions are presently essentially unknown due to the lack of experimental constraints. Only their long-range part can be constructed to some extent using SU(3) symmetry (this symmetry is partially broken), whereas the shortrange behavior is completely undetermined. We therefore neglect these interactions in this work, and are left with the nucleon-nucleon and hyperon-nucleon potentials.

Both the Paris nucleon-nucleon and the Nijmegen softcore hyperon-nucleon potentials that we use are given in the common general form 


$$
\begin{aligned}
V_{12}= & V_{C}(r)-\frac{1}{2}[\Delta \phi(r)+\phi(r) \Delta]+V_{S S}(r) \boldsymbol{\sigma}_{1} \boldsymbol{\sigma}_{2} \\
& +V_{L S}(r) \boldsymbol{L} \boldsymbol{S}+V_{Q}(r) \frac{1}{2}\left[\left(\boldsymbol{\sigma}_{1} \boldsymbol{L}\right)\left(\boldsymbol{\sigma}_{2} \boldsymbol{L}\right)+\left(\boldsymbol{\sigma}_{2} \boldsymbol{L}\right)\left(\boldsymbol{\sigma}_{1} \boldsymbol{L}\right)\right] \\
& +V_{T}(r)\left[3\left(\boldsymbol{\sigma}_{1} \hat{\boldsymbol{r}}\right)\left(\boldsymbol{\sigma}_{2} \hat{\boldsymbol{r}}\right)-\left(\boldsymbol{\sigma}_{1} \boldsymbol{\sigma}_{2}\right)\right]
\end{aligned}
$$

i.e., they contain central and momentum-dependent components as well as spin-spin, linear and quadratic spin-orbit, and tensor parts. The nucleon-nucleon $(N N)$ system may exist in two isospin states $T=0,1$, whereas the hyperonnucleon system may exist in the $T=1 / 2$ isospin state $(\Lambda N$, $\Sigma N)$ and in the $T=3 / 2$ isospin state $(\Sigma N)$. For this system, there are three couplings in the $T=1 / 2$ channel $(\Lambda \Lambda, \Lambda \Sigma$, $\Sigma \Sigma$ ) and one in the $T=3 / 2$ channel $(\Sigma \Sigma)$. When referring to couplings in the hyperon-nucleon channel, and provided there is no confusion possible, we use the notation $\Lambda \Sigma$ for denoting $\Lambda N \leftrightarrow \Sigma N$, etc. Similarly, in order to simplify the notation in the equations below, we use $\Lambda$ and $\Sigma$ for denoting the $\Lambda N$ and $\Sigma N$ channels, respectively.

Using these potentials we have to solve the BetheGoldstone $[12,13]$ integral equation in the nucleon-nucleon and hyperon-nucleon channels. We write down the equation for the later, more complex, case:

$$
\begin{aligned}
u_{Y Y^{\prime}, L L^{\prime}}(k, r)= & j_{L}(k r) \delta_{Y Y^{\prime}} \delta_{L L^{\prime}}+\frac{2}{\pi} \int_{0}^{\infty} d r^{\prime} r^{\prime 2} D_{Y Y^{\prime}, L^{\prime}}\left(r, r^{\prime}\right) \\
& \times \sum_{Y^{\prime \prime}, L^{\prime \prime}} V_{Y^{\prime} Y^{\prime \prime}, L^{\prime} L^{\prime \prime}}\left(r^{\prime}\right) u_{Y Y^{\prime \prime}, L L^{\prime \prime}}\left(k, r^{\prime}\right),
\end{aligned}
$$

with the intermediate propagator

$$
D_{Y Y^{\prime}, L^{\prime}}\left(r, r^{\prime}\right)=\int_{0}^{\infty} d k^{\prime} k^{\prime 2} \frac{j_{L^{\prime}}\left(k^{\prime} r\right) j_{L^{\prime}}\left(k^{\prime} r^{\prime}\right) f_{Y^{\prime}}\left(k^{\prime}\right)}{E_{Y}(k)-E_{Y^{\prime}}\left(k^{\prime}\right)+i \epsilon}
$$

and

$E_{Y}(k)=\frac{k_{N}^{2}}{2 M_{N}}+\frac{k_{Y}^{2}}{2 M_{Y}}+\operatorname{Re} U_{N}\left(k_{N}\right)+\operatorname{Re} U_{Y}\left(k_{Y}\right)+M_{N}+M_{Y}$.

Here $k$ and $k^{\prime}$ denote the relative momenta of the initial $N Y$ and the intermediate $N Y^{\prime}$ state, $E_{Y}(k)$ and $E_{Y^{\prime}}\left(k^{\prime}\right)$ are the corresponding energies, and $f_{Y^{\prime}}\left(k^{\prime}\right)$ denotes the angleaveraged Pauli operator in the intermediate states. More details on these quantities are given in the Appendix. In these expressions $Y, Y^{\prime}, Y^{\prime \prime}=\Lambda, \Sigma$ account for the possibility of $N \Lambda \leftrightarrow N \Sigma$ mixing through the strong interaction, and the equation has to be solved for a set of states with definite quantum numbers $T, S, J$, which have not been indicated explicitly. In practice we consider all partial waves up to $J$ $=5$. The Bethe-Goldstone equation has thus a $2 \times 2$ matrix structure due the coupling between nucleon-lambda and nucleon-sigma states, written schematically:

$$
\left(\begin{array}{c}
u_{\Lambda \Lambda} \\
u_{\Lambda \Sigma}
\end{array}\right)=\left(\begin{array}{c}
j_{L} \\
0
\end{array}\right)+\left(\begin{array}{cc}
D_{\Lambda \Lambda} V_{\Lambda \Lambda} & D_{\Lambda \Lambda} V_{\Lambda \Sigma} \\
D_{\Lambda \Sigma} V_{\Sigma \Lambda} & D_{\Lambda \Sigma} V_{\Sigma \Sigma}
\end{array}\right)\left(\begin{array}{c}
u_{\Lambda \Lambda} \\
u_{\Lambda \Sigma}
\end{array}\right)
$$

(there is another equation, obtained by interchanging $\Lambda \leftrightarrow \Sigma$ ), and a $4 \times 4$ structure when the mixing of angular momentum states through the tensor potential applies.

The solutions of the Bethe-Goldstone equation determine the diagonal $G$-matrix elements

$$
\begin{aligned}
\left\langle k_{N} k_{Y}\left|G_{Y, L}^{T S J}\right| k_{N} k_{Y}\right\rangle= & 4 \pi \int_{0}^{\infty} d r r^{2} j_{L}(k r) \\
& \times \sum_{Y^{\prime}, L^{\prime}} V_{Y Y^{\prime}, L L^{\prime}}(r) u_{Y Y^{\prime}, L L^{\prime}}(k, r),
\end{aligned}
$$

and the single-particle potentials (in the so-called continuous choice) are then given by

$$
\begin{aligned}
U_{A}^{(B)}\left(k_{A}\right)= & \sum_{T, S, J, L} \frac{(2 T+1)(2 J+1)}{\left(2 t_{A}+1\right)\left(2 s_{A}+1\right)} \\
& \times \int k_{F}^{(B)} \frac{d^{3} k_{B}}{(2 \pi)^{3}}\left\langle k_{A} k_{B}\left|G_{Y, L}^{T S J}\right| k_{A} k_{B}\right\rangle,
\end{aligned}
$$

where the notation $U_{A}^{(B)}$ denotes the single-particle potential of particle $A$ due to the interaction with particles $B$ in the medium. Carrying out the calculation for the relevant combinations $A=N, \Lambda, \Sigma$ and $B=N, \Lambda$, we obtain the total single-particle potentials of nucleons and hyperons as

$$
\begin{aligned}
& U_{N}\left(k_{N}\right)=U_{N}^{(N)}\left(k_{N}\right)+U_{N}^{(\Lambda)}\left(k_{N}\right), \\
& U_{\Lambda}\left(k_{\Lambda}\right)=U_{\Lambda}^{(N)}\left(k_{\Lambda}\right)+U_{\Lambda}^{(\Lambda)}\left(k_{\Lambda}\right), \\
& U_{\Sigma}\left(k_{\Sigma}\right)=U_{\Sigma}^{(N)}\left(k_{\Sigma}\right)+U_{\Sigma}^{(\Lambda)}\left(k_{\Sigma}\right) .
\end{aligned}
$$

(Presently we neglect the hyperon-hyperon interaction and set $U_{Y}^{(\Lambda)}=0$.) Because of the occurrence of $U_{N}$ and $U_{Y}$ in Eq. (4), the set of equations (2)-(8), together with the appropriate ones for the nucleons, constitutes a coupled system that has to be solved in a self-consistent manner.

We are interested in the total binding energy per baryon $B / A$ (the difference between total energy and energy of the interaction-free system). In the BHF approximation this quantity and the baryon number $A$ per unit volume, i.e., the baryon density $\rho$, are given by

$$
\begin{aligned}
\frac{B}{A} \rho= & \frac{4 \pi}{(2 \pi)^{3}}\left[4 \int_{0}^{k_{F}^{(N)}} d k k^{2}\left(\frac{k^{2}}{2 M_{N}}+\frac{1}{2} U_{N}(k)\right)\right. \\
& \left.+2 \int_{0}^{k_{F}^{(\Lambda)}} d k k^{2}\left(\frac{k^{2}}{2 M_{\Lambda}}+\frac{1}{2} U_{\Lambda}(k)\right)\right]
\end{aligned}
$$

and

$$
\rho=\rho_{N}+\rho_{\Lambda}=\frac{1}{3 \pi^{2}}\left(2 k_{F}^{(N) 3}+k_{F}^{(\Lambda) 3}\right) .
$$

\section{RESULTS}

\section{A. Single-particle potentials}

In order to make the subsequent discussion more transparent, we begin in Fig. 1 with a plot of the hyperon-nucleon 


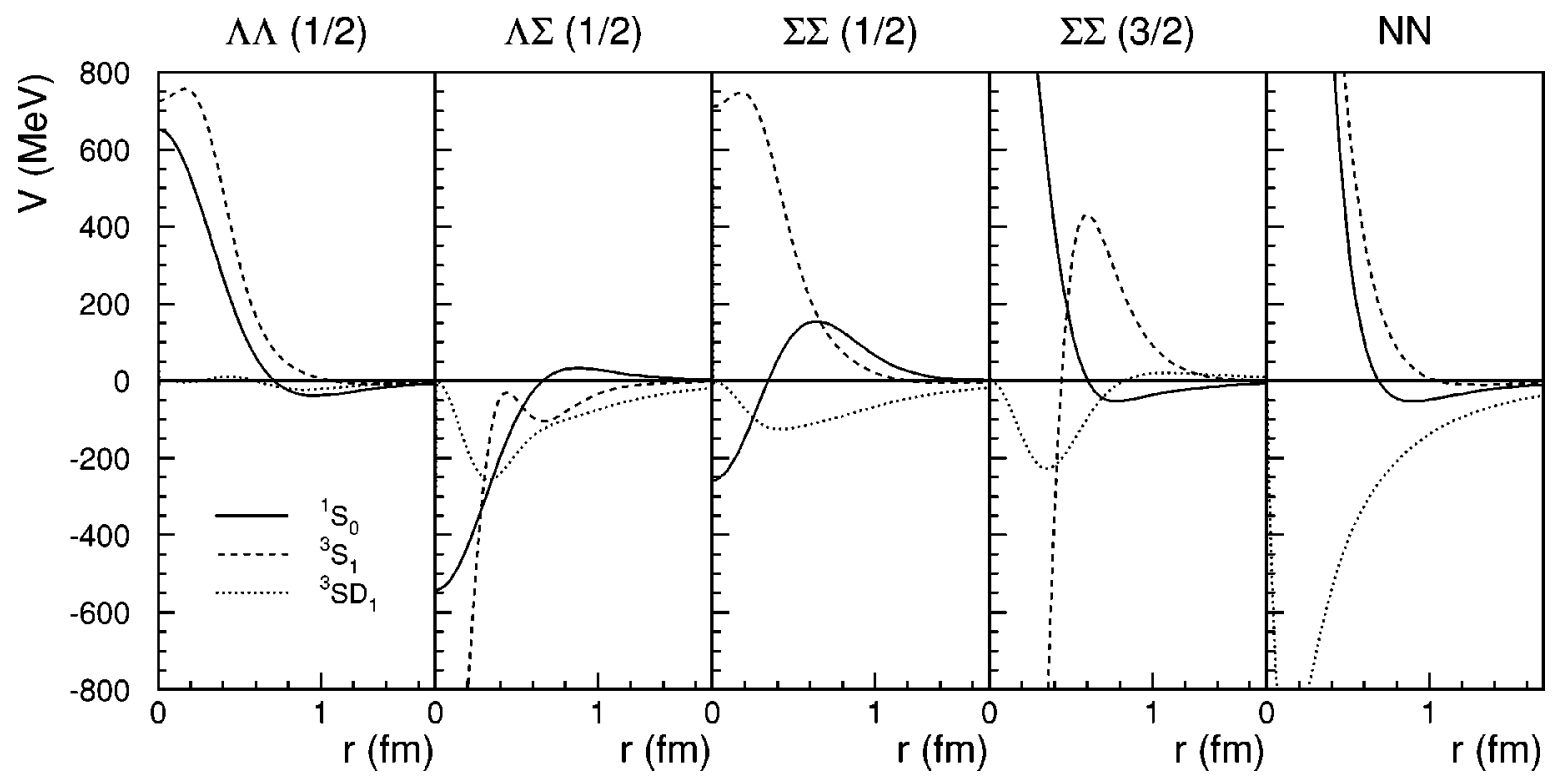

FIG. 1. The central components of the Nijmegen soft-core hyperon-nucleon potentials in the ${ }^{1} S_{0},{ }^{3} S_{1}$, and ${ }^{3} S D_{1}$ partial waves, for the four different hyperon-nucleon channel couplings. For comparison, the same components are shown for the Paris nucleon-nucleon potential on the right-hand side.

potentials in the most important partial waves ${ }^{1} S_{0},{ }^{3} S_{1}$, and ${ }^{3} S D_{1}$, and for the four couplings $\Lambda \Lambda, \Lambda \Sigma, \Sigma \Sigma(T=1 / 2)$, and $\Sigma \Sigma(T=3 / 2)$. For comparison, also the results for the Paris nucleon-nucleon potential are shown. It should be emphasized that we plot only the central components $V_{C}$ [see Eq. (1)] of the potentials, although the momentum dependent parts $(\phi)$ are known to be quite important quantitatively. Therefore only qualitative features are illustrated by the figure. We mention the following: (a) At short distances ( $r$ $\lesssim 1 \mathrm{fm}$ ) the hyperon-nucleon potentials are much "softer" and their variation is much more pronounced (nonmonotonic) than the nucleon-nucleon potential, which basically represents a monotonically rising "hard core." This implies (unfortunately) a more careful and time-consuming numerical treatment of the short-range part of the integral equations. (b) The repulsion in the hyperon-nucleon potentials (in particular the $\Sigma \Sigma$ components) appears to set in at slightly larger distances than in the nucleon-nucleon interaction. (c) There is a strong attraction for all partial waves in the "mixing', channel $\Lambda \Sigma$, in particular for the ${ }^{3} S D_{1}$ wave.

The qualitative behavior of the potentials, in particular the last point just mentioned, allows us to understand the size of the contributions of the individual partial waves to the single-particle potentials $U_{\Lambda}(k=0)$ and $U_{\Sigma}(k=0)$, which are listed in Table I for the case of individual hyperons in pure nuclear matter $\left(k_{F}^{(Y)}=0, k_{F}^{(N)}=1.35 \mathrm{fm}^{-1}\right)$. Let us re-

TABLE I. The contributions (in MeV) of various partial waves to the single-particle potentials $U_{Y}\left(k_{Y}=0\right)$ at $k_{F}^{(N)}=1.35 \mathrm{fm}{ }^{-1}$ and $k_{F}^{(\Lambda)}=0$. The total sums include partial waves up to $J=5$.

\begin{tabular}{|c|c|c|c|c|c|c|c|}
\hline \multirow[b]{2}{*}{ State } & \multicolumn{3}{|c|}{$U_{\Lambda}(0)$} & \multicolumn{4}{|c|}{$U_{\Sigma}(0)$} \\
\hline & $(\Lambda \Lambda)$ & $(\Lambda \Sigma)$ & $(\Lambda \Lambda)+(\Lambda \Sigma)$ & $(\Sigma \Sigma)(1 / 2)$ & $(\Sigma \Lambda)(1 / 2)$ & $(\Sigma \Sigma)(3 / 2)$ & $(\Sigma \Sigma)+(\Sigma \Lambda)$ \\
\hline${ }^{1} S_{0}$ & -13.0 & -2.3 & -15.3 & 7.2 & -0.5 & -12.0 & -5.2 \\
\hline${ }^{3} S S_{1}$ & 17.1 & -9.0 & 8.1 & 12.8 & -4.4 & 8.6 & 17.0 \\
\hline${ }^{3} S D_{1}$ & -1.9 & -19.2 & -21.1 & -8.4 & -14.9 & -1.9 & -25.1 \\
\hline${ }^{3} P_{0}$ & 0.4 & -0.1 & 0.2 & 2.6 & 0.0 & -2.0 & 0.6 \\
\hline${ }^{1} P_{1}$ & 2.0 & -0.4 & 1.6 & 2.0 & -0.2 & -2.7 & -0.9 \\
\hline${ }^{3} P_{1}$ & 2.2 & -0.2 & 2.0 & -5.3 & -0.0 & 6.6 & 1.3 \\
\hline${ }^{3} P P_{2}$ & -2.9 & -0.3 & -3.3 & 0.8 & -0.2 & -1.6 & -1.1 \\
\hline${ }^{3} \mathrm{PF}_{2}$ & -0.0 & -0.7 & -0.7 & -0.6 & -0.5 & -0.3 & -1.4 \\
\hline${ }^{3} D D_{1}$ & -0.06 & -0.01 & -0.07 & 0.54 & 0.00 & -0.52 & 0.02 \\
\hline${ }^{3} D S_{1}$ & -0.00 & -0.01 & -0.01 & -0.01 & 0.01 & -0.00 & -0.00 \\
\hline${ }^{1} D_{2}$ & -0.41 & -0.00 & -0.42 & 0.59 & 0.00 & -0.83 & -0.24 \\
\hline${ }^{3} D_{2}$ & -0.35 & -0.05 & -0.40 & -1.44 & 0.00 & 1.37 & -0.07 \\
\hline${ }^{3} F F_{2}$ & -0.01 & 0.00 & -0.01 & 0.09 & 0.00 & -0.10 & -0.00 \\
\hline${ }^{3} \mathrm{FP}_{2}$ & 0.00 & -0.00 & -0.00 & -0.00 & 0.00 & -0.00 & 0.00 \\
\hline Sum & & & -29.8 & & & -5.5 & -15.3 \\
\hline
\end{tabular}




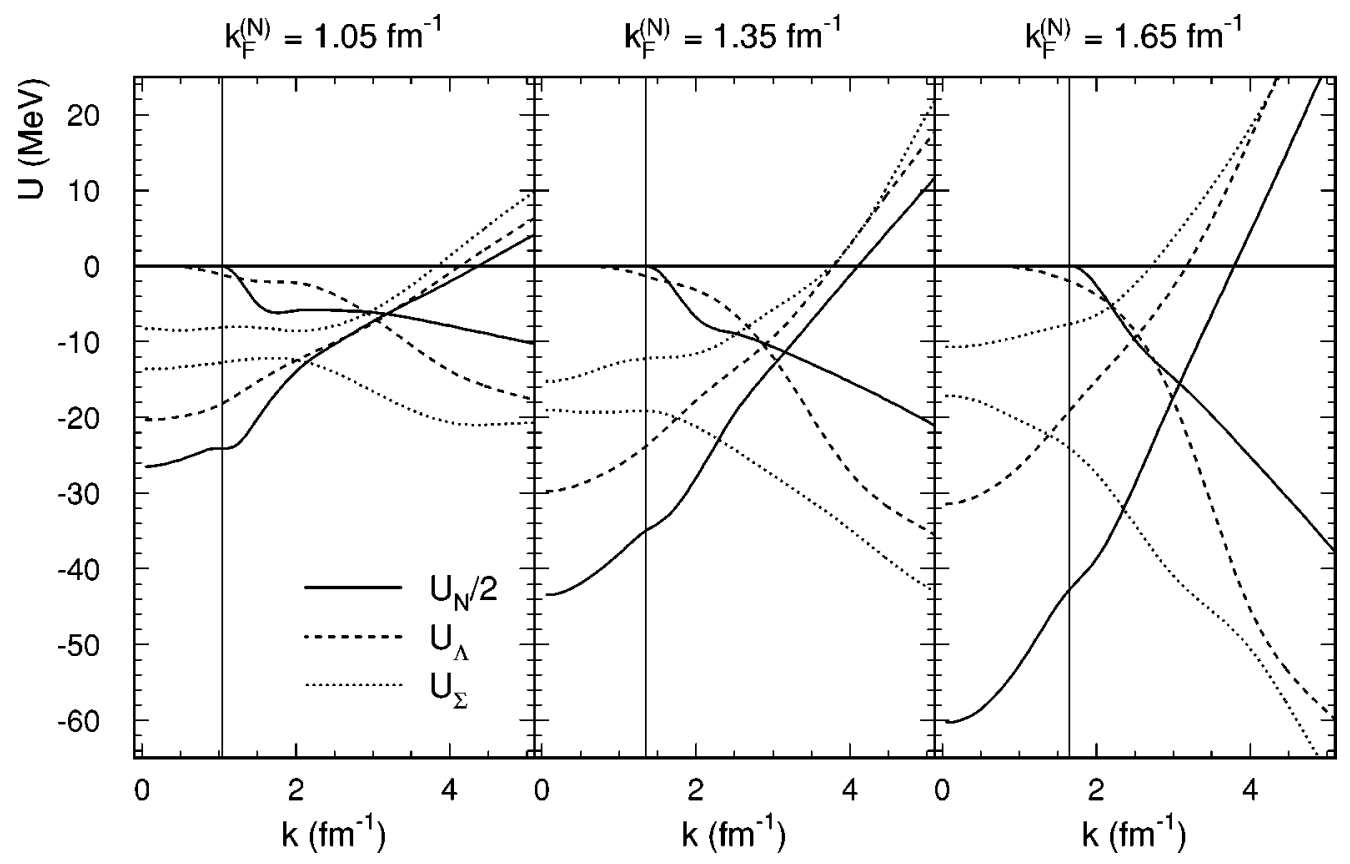

FIG. 2. Nucleon and hyperon single-particle potentials (real and imaginary parts) in pure nuclear matter $\left(k_{F}^{(\Lambda)}=0\right)$ of three different densities corresponding to $k_{F}^{(N)}=1.05,1.35,1.65 \mathrm{fm}^{-1}$. The real parts correspond to the increasing functions, whereas the imaginary parts are given by the curves with a negative slope. The nucleon curves are scaled by a factor $1 / 2$. The vertical lines denote the position of the nucleon Fermi momentum.

mind the reader that the Brueckner resummation basically cuts off the repulsive parts appearing in the potentials. For the total depth of the mean fields we obtain $U_{\Lambda}(k=0)$ $\approx-30 \mathrm{MeV}$ and $U_{\Sigma}(k=0) \approx-15 \mathrm{MeV}$, which is in the case of the lambda in good agreement with the analysis of $(\pi, K)$ and $(K, \pi)$ reactions and of hypernuclei binding energies [1], and in agreement with previous similar theoretical investigations [14-16] restricted to vanishing hyperon density. For the sigma, the experimental as well as theoretical [15] situation is less clear. Our calculation is the first one with a fully self-consistent choice of continuous singleparticle potentials, which appears to be most appropriate in particular for the sigma particle. Meson-exchange mean-field theories yield also similar results, but sometimes at the expense of adapting coupling constants [11,17-19].

In Fig. 2 we display the full momentum dependence of the single-particle potentials in pure nuclear matter at three different densities. The real nucleon single-particle potentials assume the usual momentum dependence, with a small wiggle in the curves just above the Fermi momentum, typical of first-order Brueckner calculations. The curves for the hyperon single-particle potentials have a similar shape. Their curvature is less pronounced, which indicates a smaller effective mass. Defining a global effective mass by

$$
\frac{m^{*}}{m}=\left[1+\frac{U\left(k_{F}\right)-U(0)}{k_{F}^{2} / 2 m}\right]^{-1}
$$

( $k_{F}$ is the nucleon Fermi momentum), one finds at normal nuclear matter density values of $0.69,0.84$, and 0.91 , for the nucleon, lambda, and sigma particles, respectively.

The imaginary parts of the single-particle potentials are also given in Fig. 2. For the nucleons this quantity is different from zero only when $k>k_{F}^{(N)}$ due to the complete Pauli blocking of collisions below this value. However, there is no restriction for the hyperons, since an arbitrary small excitation of the nucleon Fermi sphere can be obtained by a corresponding loss of energy of the hyperon. It is understandable that this possibility vanishes for zero-momentum $\Lambda$ particles. This is not the case for $\Sigma$ particles: The imaginary part has a nonvanishing value at zero momentum, because of the exothermic reaction $\Sigma N \rightarrow \Lambda N$. This can be checked qualitatively: In a semiclassical picture, the imaginary part is given by

$$
\operatorname{Im} U=-\left\langle\frac{k}{2 \mu} \sigma(k)\right\rangle \frac{\rho}{2},
$$

where $\mu$ is the reduced hyperon-nucleon mass [Eq. (A2)], $\sigma$ the cross section, and $\rho$ the nucleon density. An average over the Fermi sphere of the nucleon is involved, with $k$ being the hyperon-nucleon relative momentum. With the Nijmegen potential, the $\Sigma N \rightarrow \Lambda N$ cross section is roughly equal to $a / k$ [5], with $a \approx 7 \mathrm{fm}$. The above formula yields $\operatorname{Im} U \approx$ $-23 \mathrm{MeV}$ for normal density, compared to the calculated numerical value of $\approx-19 \mathrm{MeV}$. The difference can be attributed mainly to the remaining Pauli blocking that is not taken into account by the classical formula.

In Fig. 3 we show the density dependence of characteristic features of the nucleon and hyperon single-particle potentials in pure nuclear matter, namely, the depth of the mean field for a nucleon or hyperon at rest, $U(k=0)$, as well as their global effective masses, according to Eq. (11). At all densities, the binding of the hyperons is much smaller $(\$ 30 \%)$ than that of the nucleons. For all kinds of particles, the absolute value of the mean field first increases (in absolute value) as the density is increasing, reaches a maximum, and then decreases. The density at which the hyperons are 


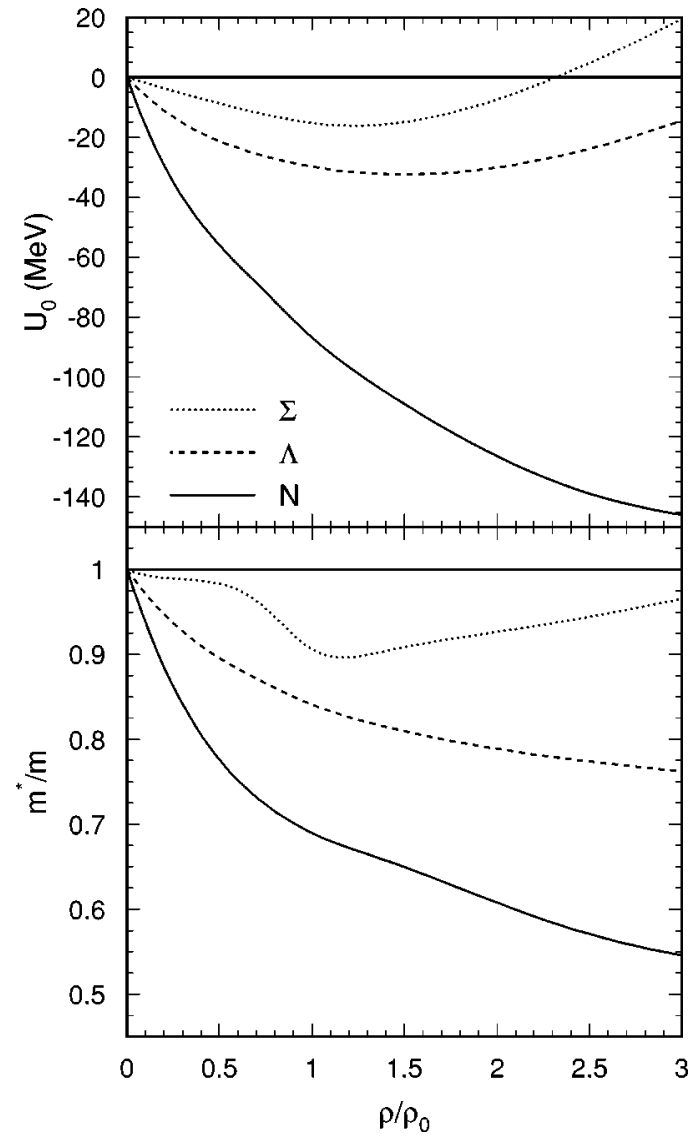

FIG. 3. Depth $U(k=0)$ of nucleon and hyperon mean fields (upper panel) and global effective masses $m * / m$ according to Eq. (11) (lower panel) in pure nuclear matter as a function of nucleon density.

most strongly bound, $\rho / \rho_{0} \approx 1.5$, is substantially smaller than the one for nucleons, $\rho / \rho_{0} \gtrsim 3$. This is due to the fact that the repulsive components of the hyperon-nucleon interaction become effective already at a larger distance than for the nucleon-nucleon one (see Fig. 1). Concerning the effective masses, their deviations from the bare values reflect the strengths of the interactions in the same manner as observed for the well depths; namely, the nucleon effective mass is smaller than the one of lambdas and sigmas, in that order. The nonmonotonic behavior with density in the case of the sigma is due to a competition between the isospin $1 / 2\left(\mathrm{~m}^{*}\right.$ $<m)$ and $3 / 2\left(m^{*}>m\right)$ contributions.

In Fig. 4 we demonstrate the effect of increasing the lambda hyperon density $\left(k_{F}^{(\Lambda)}=0.8,1.2,1.6 \mathrm{fm}^{-1}\right)$ at fixed nucleon density $\rho_{N}=\rho_{0}$. The real nucleon single-particle potential $U_{N}$ becomes increasingly deeper, compared to the pure nuclear matter case, due to the attractive hyperonnucleon interaction. This modification corresponds almost entirely to the addition of the term $U_{N}^{(\Lambda)}$ [see Eq. (8a)], whereas the modification of $U_{N}^{(N)}$ (due to the self-consistent change of the energy denominator in the Bethe-Goldstone equation) is very small. Concerning the hyperon singleparticle potentials, in our approximation scheme there is only the contribution $U_{Y}^{(N)}$ [see Eqs. (8b) and (8c)], since we neglect the hyperon-hyperon interaction. There is consequently only a small indirect effect (reduction) with increasing lambda density, via the modification of the Pauli operator and single-particle potentials in the intermediate states.

Our numerical results allow us to test the approximation

$$
\frac{U_{N}^{(\Lambda)}}{U_{\Lambda}^{(N)}} \approx \frac{\rho_{\Lambda}}{\rho_{N}}
$$

which is often used in mean-field calculations. For the three cases shown in Fig. 4 we obtain at zero momentum $(k=0)$ the ratios $U_{N}^{(\Lambda)} / U_{\Lambda}^{(N)}=0.13,0.39,0.80$, compared to $\rho_{\Lambda} / \rho_{N}$ $=0.10,0.35,0.83$, respectively. For larger momenta, the relation is less well satisfied, however.

The imaginary part of the nucleon mean field is increased, due to the possible collisions with the lambda particles. The imaginary part of the lambda potential differs from zero for $k>k_{F}^{(\Lambda)}$. This quantity remains small until $k \lesssim 2 \mathrm{fm}^{-1}$, corresponding to the opening of the $\Sigma N$ channel $\left(M_{\Sigma}-M_{\Lambda}\right.$ $\approx 78 \mathrm{MeV}$ ), to which the $\Lambda N$ is strongly coupled. It is interesting to note that the imaginary part of the $\Sigma$ singleparticle potential at low momenta is decreasing substantially (in absolute value) as the $\Lambda$ density is increasing. The reason is that the $\Sigma N \rightarrow \Lambda N$ transition is more and more hindered by the Pauli principle in the growing $\Lambda$ Fermi sphere.

\section{B. Binding energy}

The binding energy per baryon, $B / A$, as a function of baryon density $\rho=\rho_{\Lambda}+\rho_{N}$ and hyperon fraction $y=\rho_{\Lambda} / \rho$ is shown in Fig. 5. For pure nuclear matter $(y=0$, curve on the front face of the box, on the right), our calculation predicts saturation at a too large density $\left(\rho \approx 0.23 \mathrm{fm}^{-3}\right)$, although the magnitude of $B / A$ at the true saturation point $(\approx-15.1$ $\mathrm{MeV}$ ) is quite satisfactory. The failure to reproduce the correct saturation point is common to all nonrelativistic Brueckner calculations with realistic potentials. In this article we are in any case interested in the effect of adding hyperons to the system, i.e., increasing $y$. Since the binding energy gained by adding a single lambda to nuclear matter, $\left|U_{\Lambda}(0)\right|$, is larger than that by adding a nucleon, namely, $|B / A|$, it is energetically favorable (as far as the binding energy is concerned) to add hyperons to the system. Of course, when their number is increasing, the Pauli principle forces them to have kinetic energy. As a result, at some finite hyperon fraction $y_{0}$, the quantity $B / A$ displays a shallow minimum, as indicated in Fig. 5. This fraction increases with increasing baryon density, since $B / A$ decreases more and more in magnitude: Dense nuclear matter favors the binding of a larger percentage of hyperons. On the other hand, the shift of the predicted saturation point with strangeness fraction $y$ is practically negligible, as is indicated by a projected curve (nearly straight line) in the figure: The internuclear distance favored by nucleon-nucleon and hyperon-nucleon pairs is approximately the same.

It is convenient to introduce dimensionless quantities for a parametrization of the $y$ dependence of $B / A$ by a parabolic approximation:

$$
\frac{[B / A](\rho, y)}{[B / A](\rho, 0)}:=b(\rho, y) \approx 1+b_{0}-b_{0}\left(\frac{y}{y_{0}}-1\right)^{2} .
$$

The parameters $y_{0}$ and $b_{0}$, depending upon $\rho$, indicate the position of the minimum and the relative enhancement of the 


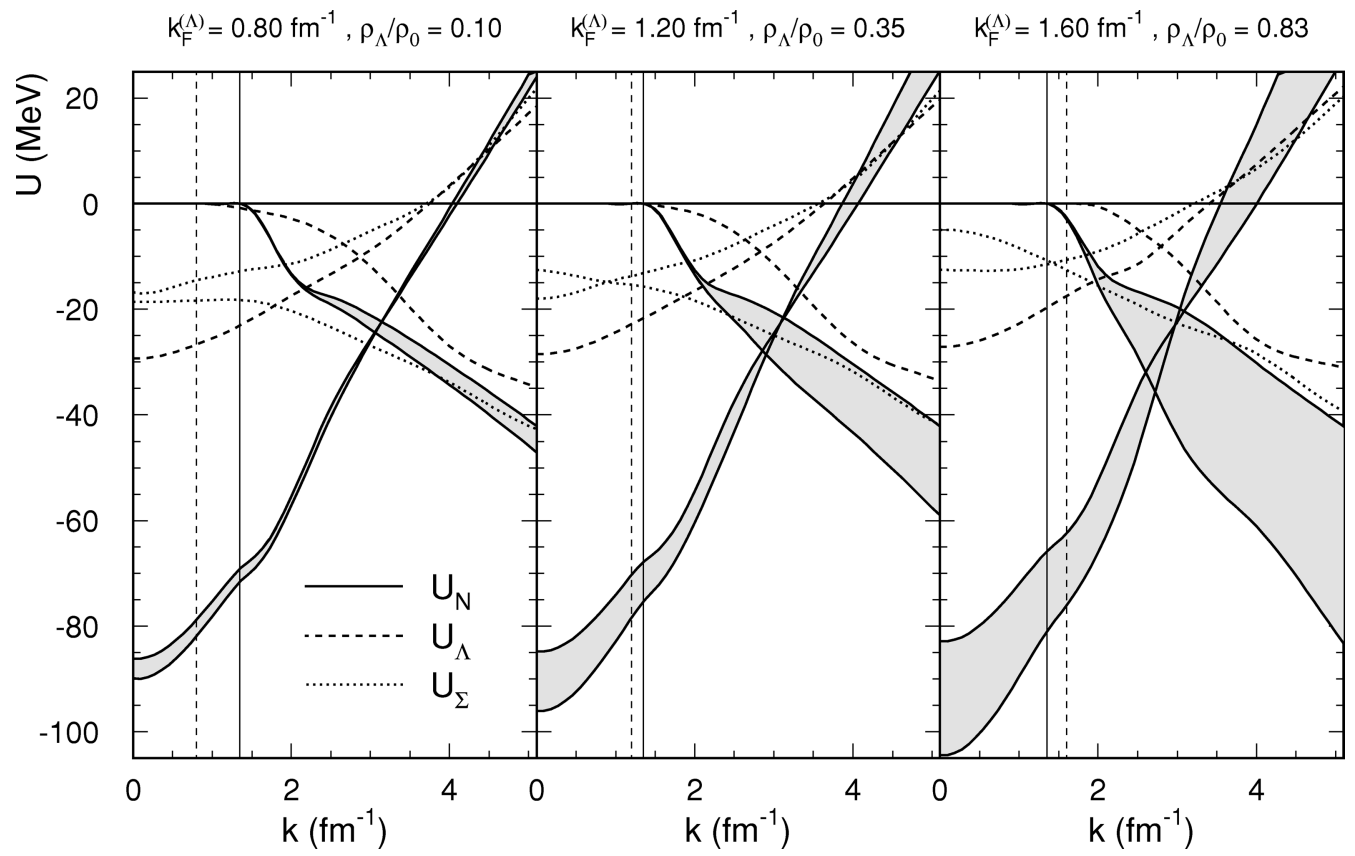

FIG. 4. Nucleon and hyperon single-particle potentials (real and imaginary parts) for three values of $k_{F}^{(\Lambda)}=0.8,1.2,1.6 \mathrm{fm}^{-1}$ at fixed $k_{F}^{(N)}=1.35 \mathrm{fm}^{-1}$. The dashed and dotted curves represent $U_{\Lambda}^{(N)}$ and $U_{\Sigma}^{(N)}$, respectively, and the solid curves $U_{N}$ (lower curves) and $U_{N}^{(N)}$ (upper curves). The shaded areas thus represent $U_{N}^{(\Lambda)}$. The real parts correspond to the increasing functions, the imaginary parts to the decreasing ones. The vertical lines denote the positions of the nucleon (solid line) and of the lambda (dashed line) Fermi momenta.

binding energy at this minimum, respectively. The function $b(\rho, y)$ for several values of the density is shown in Fig. 6 together with the dependence of the parameters $y_{0}$ and $b_{0}$ on the baryon density. The values at normal density are $y_{0} \approx 0.026$ and $b_{0} \approx 0.005$. It should be stressed, however, that the simple fit, Eq. (14), is only valid in the vicinity of the minimum $y_{0}$. For a larger hyperon fraction the binding energy $B / A$ increases faster than given by the formula above.

For a first practical application, we use our results together with a generalized Bethe-Weizsäcker formula for hypernuclear matter that was proposed in Ref. [10] in the framework of a Fermi gas model. It amounts to adding terms describing the nuclear symmetry energy, surface energy, and Coulomb energy:

$\frac{B}{A}(A, x, y)=-a_{V} b\left(\rho_{0}, y\right)+a_{x} x^{2}+\frac{a_{S}}{A^{1 / 3}}+\frac{A^{2 / 3} a_{C}}{4}(1-x-y)^{2}$,

with $A=N+Z+\Lambda, x=(N-Z) / A$, and $y=\Lambda / A$ (this is different from the definition in Ref. [10]). In the Coulomb energy term, the relation $Z / A=(1-x-y) / 2$ was used. Following Ref. [10], we choose the parameters (in MeV) $a_{V}=16$, $a_{x}=28.5, a_{S}=16.9$, and $a_{C}=0.72$. The minimum of this expression with respect to $x$ and $y$ for fixed $A$ defines the line of maximum binding for hypernuclei, $(x, y)_{\min }(A)$. Assuming the parametrization, Eq. (14), one finds, for $x_{\min }, y_{\min }$, and the proton fraction $(Z / A)_{\min }$,

$$
x_{\min }=\frac{1-y_{0}}{1+\frac{a_{x} y_{0}^{2}}{a_{V} b_{0}}+\frac{4 a_{x}}{A^{2 / 3} a_{C}}},
$$

$$
\begin{aligned}
& y_{\min }=y_{0}+\frac{a_{x} y_{0}^{2}}{a_{V} b_{0}} x_{\min }, \\
& (Z / A)_{\min }=\frac{2 a_{x}}{A^{2 / 3} a_{C}} x_{\min } .
\end{aligned}
$$

Were it not for the Coulomb term $\left(a_{C}=0\right), y_{\min }$ would coincide with the value $y_{0} \approx 0.026$ determined earlier. The Cou-

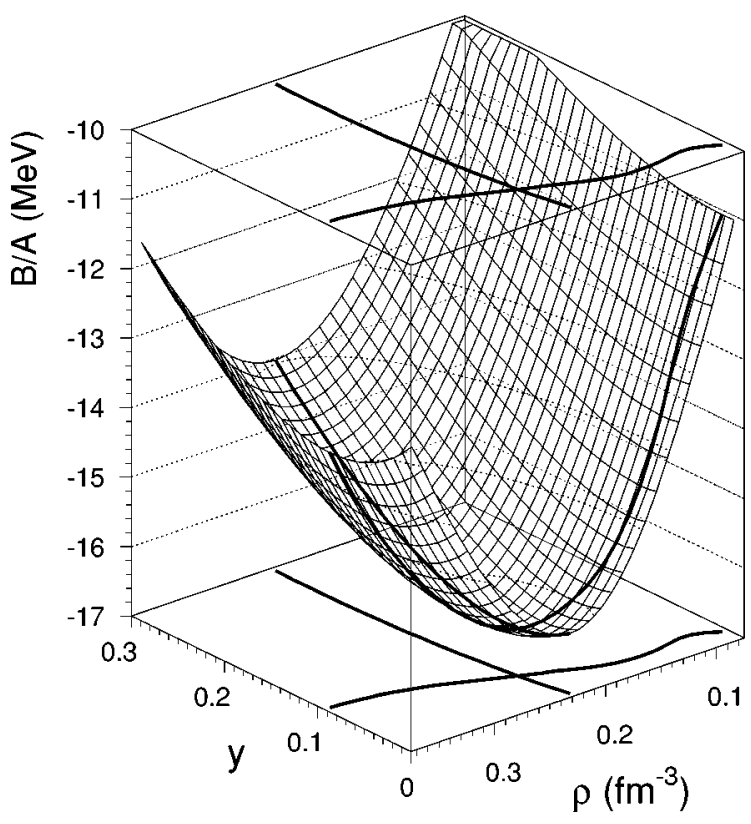

FIG. 5. Binding energy per baryon of hyperonic nuclear matter as a function of baryon density $\rho=\rho_{\Lambda}+\rho_{N}$ and lambda fraction $y$ $=\rho_{\Lambda} / \rho$. The projected curves show the locations of the minima for fixed $y$ or $\rho$, respectively. 


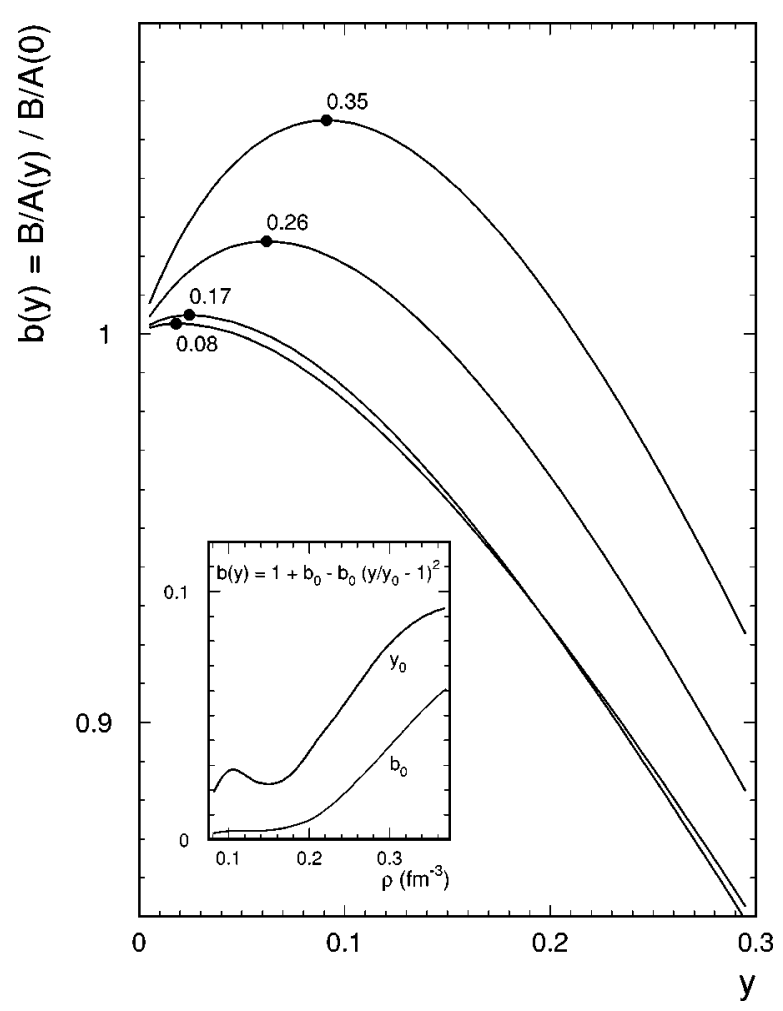

FIG. 6. Normalized binding energy per baryon as a function of lambda fraction $y$ for fixed values of the baryon density $\rho$ [indicated by the numbers near the curves $\left(\mathrm{in}^{-3}\right)$ ]. The positions of the maxima are marked. The inset shows the position $y_{0}$ of the minimum and the relative enhancement of binding energy $b_{0}$, Eq. (14), as functions of the baryon density.

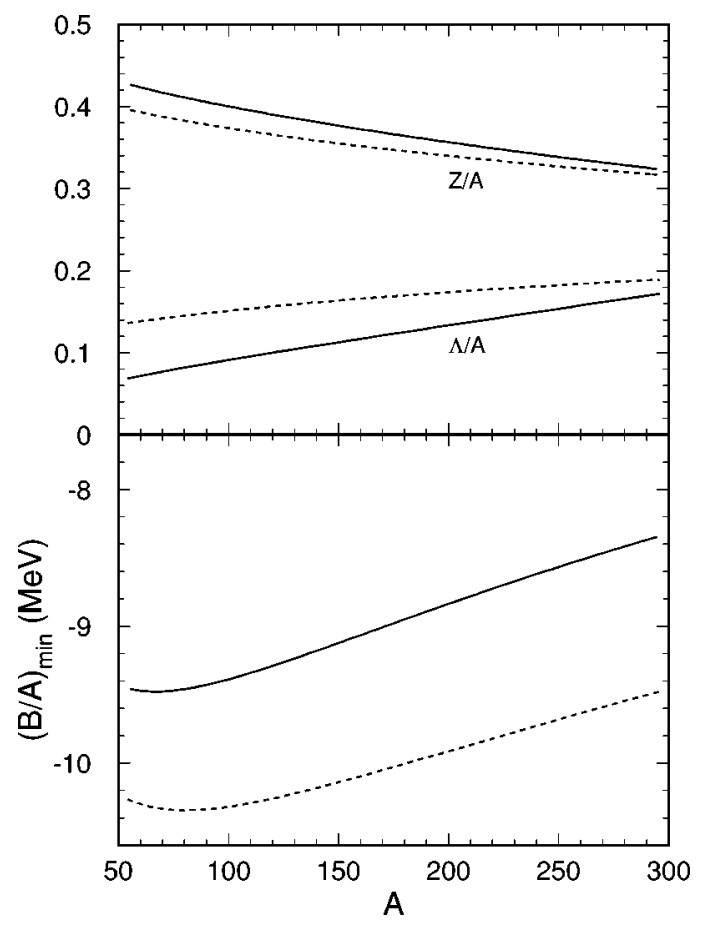

FIG. 7. Charge fraction $Z / A$, hyperon fraction $\Lambda / A$, and binding energy per baryon as functions of mass number $A$, as predicted by the generalized mass formula Eq. (15) (solid lines). The dashed curves show the results of Ref. [10].

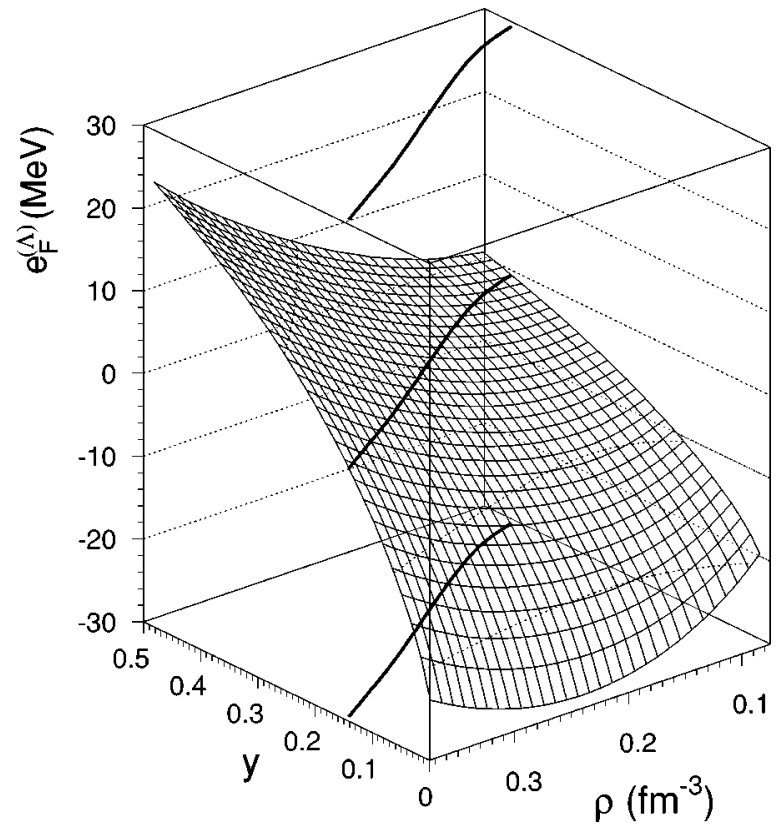

FIG. 8. Fermi energy of the lambda hyperon as a function of baryon density $\rho$ and lambda fraction $y$. The projected curve shows the location of vanishing Fermi energy.

lomb repulsion, however, favors the replacement of protons with lambdas, leading to an upward shift of $y_{\min }$, increasing with the mass number $A$. This can easily be checked on the above formulas. It is also seen in Fig. 7, where we plot the dependence of $y_{\min }$ and $(Z / A)_{\min }$ on $A$, together with the binding energy $(B / A)_{\min }$ at this point. For large mass numbers $A \approx 300$, our results are very similar to those of Ref. [10] (which are displayed as well in the figure), whereas we find a substantially smaller hyperon fraction at small $A \approx 100$. The reason is the assumption of momentum-independent hyperon-nucleon mean fields $U_{\Lambda}^{(N)}=-28 \mathrm{MeV}$ and $U_{N}^{(\Lambda)}=$ $-14 \mathrm{MeV}$ made in that reference, which is not confirmed by our calculation: At the Fermi momentum the singleparticle potentials are substantially smaller than at zero momentum; see Fig. 2. We therefore obtain less attraction than in Ref. [10]. [The approximation $b\left(\rho_{0}, y\right)=1+\alpha y$ $-\beta y^{2}, \alpha=0.71, \beta=3.55$ was derived in that work.]

The previous considerations dealt with the mostly bound hypernuclei. Another related problem is the maximum strangeness that hypernuclei of given baryon number can sustain. This limit corresponds to the hyperon drip line. Beyond this line, the last hyperon would be unbound. For actual hypernuclei, the determination of this line would require detailed calculations. To have a rough idea, we looked at the maximum hyperon fraction for which the infinite system is still bound. The latter is determined by a vanishing hyperon Fermi energy $e_{F}^{(\Lambda)}$. The results are given in Fig. 8. We find that the maximum lambda fraction at the nuclear matter density is about 0.4 , and that it decreases with increasing baryon density. This is due to the fact that with rising baryon density the kinetic part of the lambda Fermi energy becomes more and more dominant compared to the mean-field contribution.

Of course, the previous results are not to be taken too seriously, because the hyperon-hyperon interaction is neglected and, related to that, the important phenomenon of $\Xi$ condensation is disregarded. Rather, they provide a lower 
bound on the strangeness fraction in hypernuclei and demonstrate the importance of a truly microscopic treatment for quantitative predictions.

\section{CONCLUSIONS}

We presented in this work a detailed microscopic calculation of the hyperon (lambda) symmetry energy in nuclear matter, i.e., the change of binding energy when replacing nucleons by hyperons. Our theoretical framework is the selfconsistent nonrelativistic Brueckner-Hartree-Fock approximation with the continuous choice of single-particle potentials, which is expected to work well for moderate densities $\rho / \rho_{0} \lesssim 2, \ldots, 3$. This is indeed the case for pure nuclear matter. To obtain a refined agreement, like saturation at the correct density, requires adding contributions like third-order graphs in the hole-line expansion [20,21] (their numerical importance is still an unsettled issue, even for pure nuclear matter). At higher densities, besides the increasing relevance of higher order nucleonic diagrams, internal (quark) degrees of freedom should progressively become important, and a relativistic treatment appears more appropriate. Nevertheless, the hyperon (symmetry) energy should not be very sensitive to the slightly imperfect description of pure nuclear matter. Furthermore, the adapted framework, with the so-called continuous choice, allows a reliable determination of the singleparticle potentials, including their imaginary parts.

We have used one of the most advanced microscopic hyperon-nucleon interactions. Unfortunately, we were forced to neglect the hyperon-hyperon interaction, in view of the insufficient knowledge of the latter. For this reason, the results presented here are restricted to small values of the hyperon fraction. We have carried out some exploratory calculations based on simple guesses for the $\Lambda-\Lambda$ potential, but found that the results depend very strongly on the shortrange behavior of the interaction that is not controlled by symmetry considerations. Note that it is not even clear that the $\Lambda-\Lambda$ potential is repulsive. According to Ref. [2], the analysis of some double $\Lambda$ hypernuclei would suggest that the $\Lambda-\Lambda$ potential yields as much binding as the $\Lambda-N$ potential at low energy. For the time being, without sufficient restrictions by experimental data, we consider such an extension therefore as premature.

The results obtained in this work are suitable as a foundation for Thomas-Fermi calculations of heavy hypernuclei $[18,19]$. We intend to pursue such applications, as well as an extension of our formalism to hyperonic neutron matter. This is of particular interest for the physics of neutron stars, where the condensation of hyperons (most importantly $\Sigma^{-}$and $\Lambda$ ) might have important effects on the equation of state and therefore possibly on global observables of the star [4].

\section{ACKNOWLEDGMENTS}

We would like to thank A. Kerman and A. Ramos for interesting discussions and T. Rijken for a helpful communication.

\section{APPENDIX}

\section{Kinematics}

The energy of the nucleon-hyperon pair $N Y$ can be written in terms of total and relative momenta $\boldsymbol{p}$ and $\boldsymbol{k}$, respectively:

$$
E_{Y}(p, k)=\frac{p^{2}}{2 M}+\frac{k^{2}}{2 \mu}+\operatorname{Re} U_{N}\left(k_{N}\right)+\operatorname{Re} U_{Y}\left(k_{Y}\right)+M_{N}+M_{Y},
$$

$$
\begin{gathered}
M=M_{N}+M_{Y}, \quad \mu=\frac{M_{N} M_{Y}}{M_{N}+M_{Y}}, \\
\boldsymbol{k}_{Y, N}=\boldsymbol{p}_{Y, N} \pm \boldsymbol{k}, \quad \boldsymbol{p}_{Y, N}=\frac{M_{Y, N}}{M} \boldsymbol{p} .
\end{gathered}
$$

\section{Pauli operator and energy denominator}

Because of the presence of two different Fermi seas in the intermediate state, there are two restrictions on the angle $\cos \theta: \equiv z=\hat{\boldsymbol{p}} \hat{\boldsymbol{k}}$, namely,

$$
\begin{aligned}
& z>-z_{Y}=-\frac{p_{Y}^{2}+k^{2}-k_{F}^{(Y) 2}}{2 p_{Y} k}, \\
& z<+z_{N}=+\frac{p_{N}^{2}+k^{2}-k_{F}^{(N) 2}}{2 p_{N} k} .
\end{aligned}
$$

Taking account of all possible cases of vanishing, complete, and partial blocking of the two species, and introducing the notation $[a|b| c]: \equiv \max (a, \min (b, c))$, the angle-averaged Pauli operator can be written in the following compact form:

$$
f_{Y}(p, k)=\left[0\left|\frac{1}{2}\left(\left[-1\left|z_{N}\right| 1\right]+\left[-1\left|z_{Y}\right| 1\right]\right)\right| 1\right] .
$$

The energy denominator in Eq. (3) also depends, via the single-particle potentials, on the variable $z$ that is restricted to a certain interval by the Pauli operator in the numerator. In the spirit of Brueckner's original approach [12], we carry out an angle averaging by replacing $z$ with its angle-averaged value, namely,

$$
\langle z\rangle=\frac{1}{2}\left(\left[-1\left|z_{N}\right| 1\right]-\left[-1\left|z_{Y}\right| 1\right]\right),
$$

using the notation introduced above. (In the case of symmetric nuclear matter, the energy denominator does not contain a term linear in $z$ due to identical single-particle potentials. The angle averaging of $z^{2}$ leads in that case to the prescription $\left\langle z^{2}\right\rangle=f^{2} / 3$.)

\section{Momentum-dependent potential}

The Bethe-Goldstone equation for the relative correlated wave function $u$ reads

$$
u\left(r^{\prime}\right)=j_{l}\left(k r^{\prime}\right)+\frac{2}{\pi} \int_{0}^{\infty} d r r^{2} D_{l}\left(r^{\prime}, r\right)\left[V_{l}(r) u(r)\right] .
$$

For a momentum-dependent potential of the form

$$
V_{l}(r)=-\frac{1}{2}\left[\Delta_{l} \phi(r)+\phi(r) \Delta_{l}\right], \quad \Delta_{l}=\partial_{r}^{2}+\frac{2}{r} \partial_{r}-\frac{l(l+1)}{r^{2}}
$$


it can (in order to avoid computing second derivatives of the wave function) be converted by partial integration into [20]

$$
\begin{aligned}
u\left(r^{\prime}\right)= & j_{l}\left(k r^{\prime}\right)+\frac{2}{\pi} \int_{0}^{\infty} d r r^{2}\left\{\left[F_{l}\left(r^{\prime}, r\right) \phi(r)+D_{l}\left(r^{\prime}, r\right)\right.\right. \\
& \left.\left.\times\left(\frac{\phi^{\prime}(r)}{r}+\frac{\phi^{\prime \prime}(r)}{2}\right)\right] u(r)+\left[D_{l}\left(r^{\prime}, r\right) \phi^{\prime}(r)\right] u^{\prime}(r)\right\},
\end{aligned}
$$

with the Green functions

$$
\begin{aligned}
& D_{l}\left(r, r^{\prime}\right)=\int_{0}^{\infty} d k k^{2} \frac{j_{l}(k r) j_{l}\left(k r^{\prime}\right) f(k)}{W-E(k)+i \epsilon}, \\
& F_{l}\left(r, r^{\prime}\right)=\int_{0}^{\infty} d k k^{4} \frac{j_{l}(k r) j_{l}\left(k r^{\prime}\right) f(k)}{W-E(k)+i \epsilon} .
\end{aligned}
$$

The derivatives of the potential, $\phi^{\prime}$ and $\phi^{\prime \prime}$, can be performed analytically. For the sake of numerical evaluation, it is convenient to improve the rate of convergence of the above integrals by adding and subtracting suitable terms. Splitting into real and imaginary parts, one obtains

$$
\begin{aligned}
\operatorname{Re} D_{l}\left(r, r^{\prime}\right)= & \mathcal{P} \int_{0}^{k_{\max }} d k j_{l}(k r) j_{l}\left(k r^{\prime}\right)\left(\frac{k^{2} f(k)}{W-E(k)}+2 \mu\right) \\
& -\frac{\pi \mu}{2 l+1} \frac{\min \left(r, r^{\prime}\right)^{l}}{\max \left(r, r^{\prime}\right)^{l+1}},
\end{aligned}
$$

$$
\operatorname{Im} D_{l}\left(r, r^{\prime}\right)=-\pi k_{0}\left|\mu_{0}^{*}\right| j_{l}\left(k_{0} r\right) j_{l}\left(k_{0} r^{\prime}\right) f\left(k_{0}\right),
$$

$$
\begin{aligned}
\operatorname{Re} F_{l}\left(r, r^{\prime}\right)= & \mathcal{P} \int_{0}^{k_{\max }} d k k^{2} j_{l}(k r) j_{l}\left(k r^{\prime}\right)\left(\frac{k^{2} f(k)}{W-E(k)}+2 \mu\right) \\
& -\pi \mu \frac{\delta\left(r-r^{\prime}\right)}{r^{2}}, \\
& \operatorname{Im} F_{l}\left(r, r^{\prime}\right)=k_{0}^{2} \operatorname{Im} D_{l}\left(r, r^{\prime}\right),
\end{aligned}
$$

with $\mu$ being the reduced mass of the intermediate state [Eq. (A2)], $k_{0}$ denoting the zero of the energy denominator, $E\left(k_{0}\right)=W$, and $\mu_{0}^{*}=\left[\left.2\left(\partial E(k) / \partial k^{2}\right)\right|_{k_{0}}\right]^{-1}$ the effective reduced mass of the intermediate two-particle system at this point. In these equations, the symbol $\mathcal{P}$ stands for the principal part of the integral and $k_{\max }$ corresponds to a numerical cutoff. It should be chosen large enough, such that the result is sufficiently insensitive to it. In practice, a value of $20 \mathrm{fm}^{-1}$ is more than enough.

The $G$-matrix elements are finally obtained by

$$
\begin{aligned}
\left\langle k\left|G_{l}\right| k\right\rangle= & 4 \pi \int_{0}^{\infty} d r r^{2} j_{l}(k r)\left[V_{l}(r) u_{l}(k, r)\right] \\
= & 4 \pi \int_{0}^{r_{\max }} d r r^{2}\left[j _ { l } ( k r ) \left(k^{2} \phi(r)-(l+1) \frac{\phi^{\prime}(r)}{r}\right.\right. \\
& \left.\left.-\frac{\phi^{\prime \prime}(r)}{2}\right)+j_{l+1}(k r) k \phi^{\prime}(r)\right] u_{l}(k, r)
\end{aligned}
$$

with a cutoff $r_{\max } \approx 10 \mathrm{fm}$, which is also used in the discretization and numerical solution of the Bethe-Goldstone equation (A9).
[1] C. B. Dover and A. Gal, Prog. Part. Nucl. Phys. 12, 171 (1984); D. J. Millener, C. B. Dover, and A. Gal, Phys. Rev. C 38, 2700 (1988); C. B. Dover, D. J. Millener, and A. Gal, Phys. Rep. 184, 1 (1989); R. E. Chrien and C. B. Dover, Annu. Rev. Nucl. Part. Sci. 39, 113 (1989); H. Feshbach, Nucl. Phys. A507, 219c (1990).

[2] S. Aoki et al., Prog. Theor. Phys. 85, 128 (1991); C. B. Dover, D. J. Millener, A. Gal, and D. H. Davis, Phys. Rev. C 44, 1905 (1991); B. F. Gibson, in Properties and Interactions of Hyperons, edited by B. F. Gibson, P. D. Barnes, and K. Nakai (World Scientific, Singapore, 1994), p. 55; J. B. Franklin, Nucl. Phys. A585, 83c (1995).

[3] A. J. Baltz, C. B. Dover, S. H. Kahana, Y. Pang, T. J. Schlagel, and E. Schnedermann, Phys. Lett. B 325, 7 (1994).

[4] A. G. W. Cameron, Astrophys. J. 130, 884 (1959); V. R. Pandharipande, Nucl. Phys. A178, 123 (1971); H. A. Bethe and M. B. Johnson, ibid. A230, 1 (1974); N. K. Glendenning, ibid. A493, 521 (1989); G. Baym, ibid. A590, 233c (1995).

[5] P. Maessen, Th. Rijken, and J. de Swart, Phys. Rev. C 40, 2226 (1989).

[6] A. Reuber, K. Holinde, and J. Speth, Nucl. Phys. A570, 543 (1994).

[7] J. K. Ahn et al., Nucl. Phys. A585, 165c (1995).
[8] M. Lacombe, B. Loiseau, J. M. Richard, R. Vinh Mau, J. Côté, P. Pirès, and R. de Tourreil, Phys. Rev. C 21, 861 (1980).

[9] H.-J. Schulze, A. Lejeune, J. Cugnon, M. Baldo, and U. Lombardo, Phys. Lett. B 355, 21 (1995).

[10] C. B. Dover and A. Gal, Nucl. Phys. A560, 559c (1993).

[11] J. Schaffner, C. Greiner, and H. Stöcker, Phys. Rev. C 46, 322 (1992); J. Schaffner, C. B. Dover, A. Gal, C. Greiner, D. J. Millener, and H. Stöcker, Ann. Phys. (N.Y.) 235, 35 (1994).

[12] K. A. Brueckner and J. L. Gammel, Phys. Rev. 109, 1023 (1958).

[13] J.-P. Jeukenne, A. Lejeune, and C. Mahaux, Phys. Rep., Phys. Lett. 25C, 83 (1976).

[14] J. Rożynek and J. Dạbrowski, Phys. Rev. C 20, 1612 (1979).

[15] Y. Yamamoto and H. Bando, Prog. Theor. Phys. 83, 254 (1990); Y. Yamamoto, T. Motoba, H. Himeno, K. Ikeda, and S. Nagata, Prog. Theor. Phys. Suppl. 117, 361 (1994).

[16] J. Hao, T. Kuo, A. Reuber, K. Holinde, J. Speth, and D. J. Millener, Phys. Rev. Lett. 71, 1498 (1993); D. Halderson, Phys. Rev. C 48, 581 (1993); M. Hjorth-Jensen, A. Polls, A. Ramos, and H. Müther, Nucl. Phys. A605, 458 (1996).

[17] N. K. Glendenning, Z. Phys. A 326, 57 (1987).

[18] J. Mareš and J. Žofka, Z. Phys. A 333, 209 (1989); 345, 47 (1993). 
[19] M. Rufa, J. Schaffner, J. Maruhn, H. Stöcker, W. Greiner, and P.-G. Reinhard, Phys. Rev. C 42, 2469 (1990).

[20] A. Lejeune, P. Grangé, M. Martzolff, and J. Cugnon, Nucl. Phys. A453, 189 (1986).
[21] B. D. Day, Phys. Rev. C 24, 1203 (1981); B. D. Day and R. B. Wiringa, ibid. 32, 1057 (1985); M. Baldo, I. Bombaci, G. Giansiracusa, U. Lombardo, C. Mahaux, and R. Sartor, ibid. 41, 1748 (1990). 